\title{
Comportamiento de híbridos de maíz amarillo duro en la localidad de La Molina, Perú
}

\author{
Behavior of yellow corn hybrids in town of La Molina, Perú \\ Julián Chura Chuquija ${ }^{1}$ y Jorge Tejada Soraluz ${ }^{1 *}$
}

\begin{abstract}
RESUMEN
El maíz amarillo duro es el cultivo de mayor producción mundial, principal insumo en la elaboración de alimento para aves y fuente de empleo permanente. Se evaluaron 16 híbridos de maíz amarillo duro en el año 2010, con el objetivo de determinar los más prometedores en cuanto a rendimiento y otras características deseables. Respecto de rendimiento de grano los mejores híbridos fueron: D-8008, BF-9417, BF-9719, BG-9619, E-8008, C-8008 y BE-9005 con 10.9, 10.8, 10.6, 10.5, $10.3,9.8$ y 9.5 t/ ha, respectivamente. La menor altura de planta fue para Star y M-8480 con 1,39 m y 1,32 m, respectivamente. Híbridos precoces fueron M-8480, Maximus, BG-9621 y BF-9302 con 97, 96, 95 y 94 días a la floración masculina. El híbrido con mayor peso de 100 granos fue BG-9621 con 36,5 g. La mayor longitud de mazorca fue para el híbrido BE-9005 con 16,7 cm. P30F35 obtuvo el mayor diámetro de mazorca con 5,09 cm. El mayor número de hileras fue para el híbrido BF-9719 con 17,3. El mayor número de granos por hilera lo obtuvo el híbrido BG-9619 con 31,8. Uno de los híbridos con características más favorables fue BF-9417, que obtuvo un rendimiento de 10,81 t/ha, 165,28 cm de altura de planta, 104,25 días a la floración masculina, 14,17 cm de longitud de mazorca, 16,75 hileras y 29,58 granos por hilera.
\end{abstract}

Palabras clave: maíz híbrido, rendimiento, caracteres biométricos.

\begin{abstract}
Yellow corn is the largest crop of world production. It is the main input in the production of food for poultry and source of permanent employment. Sixteen yellow corn hybrids were evaluated in 2010, with the objective of determining the most promising hybrids in terms of yield and other desirable characteristics. The highest grain yields were obtained with D-8008, BF-9417, BF-9719, BG-9619, E-8008, C-8008 and BE-9005 with 10.9, 10.8, 10.6, 10.5, 10.3, 9.8 and 9.5 t/ha, respectively. Plant height was lower for Star and M-8480 with 1.39 m and $1.32 \mathrm{~m}$, respectively. Early hybrids were M-8480, Maximus, BG-9621 and BF-9302 with 97 , 96, 95 and 94 days to male flowering. The hybrid with the greatest weight (36.5 g) of 100 grains was BG-9621. The largest ear $(16.7 \mathrm{~cm})$ was obtained with BE-9005. P30F35 had the largest ear diameter $(5.09 \mathrm{~cm})$. The largest number of ear rows $(17.3)$ was present in BF-9719. The largest number of kernels per row (31.8) was found in BG-9619. One of the hybrids with more favorable characteristics was BF-9417: 10.81 t/ha, $165.28 \mathrm{~cm}$ plant height, 104.25 days to male flowering, ear length was $14.18 \mathrm{~cm}, 16.75$ ear rows and 29.58 kernels per row.
\end{abstract}

Key words: hybrid corn, yield, biometrical characters.

\section{Introducción}

A nivel mundial el maíz es uno de los tres cereales más importantes y antiguos que se conoce. En producción, en 2012 ocupó el primer lugar a nivel mundial, seguido del arroz cáscara y trigo (Organización de las Naciones Unidas para la Alimentación y la Agricultura, 2013). En Perú es un cultivo importante, ya que sirve como materia prima en la elaboración de alimento para aves y cerdos, es fuente de empleo permanente (Ministerio de
Agricultura OGPA-DGPA, 2011) y su participación en el PBI agropecuario de 2011 fue 2,6\% (Ministerio de Agricultura, 2012). Además presenta bajo contenido de aflatoxinas, que son micotoxinas producidas por hongos del género Aspergillus, lo que ocasiona menor mortandad en aves (Ministerio de Agricultura OGPA-DGPA, 2011).

El uso de maíces híbridos constituye un paso importante para el desarrollo de la agricultura, siendo considerado una de las mejores innovaciones en el fitomejoramiento (Paliwal, 2001). La siembra de

\footnotetext{
1 Universidad Nacional Agraria La Molina, Ap. 456, Lima, Perú.

* Autor por correspondencia: jorgetejada@ lamolina.edu.pe
} 
híbridos, el nitrógeno y densidades más altas de plantas incrementaron los rendimientos de maíz en EE.UU. en 7,85 t/ha por año desde 1950 a 1970 (Jugenheimer, 1981).

La principal estrategia para lograr el autoabastecimiento de maíz amarillo es el aumento de la productividad (Sevilla, 2000), lo que implica utilizar semillas de buena calidad de maíces híbridos con alto potencial de rendimiento (Salhuana y Scheuch, 2004; Vásquez et al., 2003), los que deben ser evaluados en diferentes localidades, fechas de siembra, densidades, etc., ya que los rendimientos pueden variar con diferente manejo y ambientes (Nakasone, 1996).

Los componentes del rendimiento del maíz están determinados por características biométricas de la mazorca (longitud y diámetro de la mazorca, número de hileras y número de granos por hilera), número de mazorcas por planta, peso de 1000 granos, etc. Diversos estudios así lo corroboran, sobre todo los realizados por Ferraris y Couretot (2004), quienes evaluaron el comportamiento de 26 híbridos de maíz y los siguientes componentes del rendimiento: número de granos $/ \mathrm{m}^{2}$, peso de 1000 granos y número de hileras por mazorca. Rivetti (2006) luego de evaluar el rendimiento de grano y sus componentes bajo diferentes regímenes de riego, estableció los siguientes componentes del rendimiento: número de hileras por mazorca, número de granos $/ \mathrm{m}^{2}$, número de granos por hilera y el peso de 1000 granos.

Características deseables en plantas de maíz son, principalmente, poca altura de planta, mayor precocidad y hojas superiores erectas (ángulo de inserción de hoja pequeño). En la costa peruana, por ejemplo, el sistema de producción requiere híbridos de maíz más precoces y de porte más bajo, que puedan sembrarse a altas densidades (Chura y Sevilla, 2002). Asimismo, hojas superiores erectas combinadas con hojas horizontales inferiores dan lugar a un uso más eficiente de la radiación por parte de la capa total de hojas (Paliwal, 2001).

El objetivo del presente estudio fue determinar los híbridos de maíz amarillo duro más prometedores, respecto del rendimiento de grano, sus componentes y otras características deseables.

\section{Materiales y Métodos}

El ensayo se realizó en el Campo Agrícola Experimental de la Universidad Nacional Agraria
La Molina, Lima, Perú, ubicada a $12^{\circ} 05^{\prime} 06^{\prime \prime} \mathrm{S}$, $76^{\circ} 57^{\prime} 06^{\prime \prime} \mathrm{W}$ y una altitud de $251 \mathrm{~m}$. El material experimental estuvo formado por 16 híbridos de maíz amarillo duro, 10 de ellos fueron experimentales y 6 comerciales.

El suelo fue franco arenoso y tanto la salinidad $(1,00 \mathrm{dS} / \mathrm{m})$ como el $\mathrm{pH}(7,5)$ no fueron limitante para el cultivo (Beingolea et al., 1993). Durante el ensayo, la temperatura mínima promedio fue $14,21^{\circ} \mathrm{C}$ y la temperatura máxima promedio fue $21,18^{\circ} \mathrm{C}$, no siendo impedimento para el normal crecimiento y desarrollo del cultivo.

El manejo agronómico fue similar a un campo comercial de maíz amarillo, sembrándose el 16 de abril de 2010 y cosechándose el 15 de noviembre del mismo año. Se realizaron oportunamente las aplicaciones de plaguicidas, riegos, fertilización, cultivo y aporque. La siembra se hizo a una distancia entre surcos de $85 \mathrm{~cm}$ y $32 \mathrm{~cm}$ entre golpes ( 2 semillas/golpe), teniendo una densidad de 73520 plantas/ha. El área del ensayo fue $1114,24 \mathrm{~m}^{2}$, dividida en 4 bloques de 16 parcelas de 17,41 $\mathrm{m}^{2}$ cada una.

Se evaluó la altura de planta, altura de mazorca, ángulo de inserción de hoja, días a la floración femenina, días a la floración masculina, rendimiento de grano, humedad del grano, peso de diez mazorcas, peso de grano de diez mazorcas, peso de cien granos, índice de mazorca, porcentaje de desgrane, longitud de mazorca, diámetro de la mazorca, número de hileras y número de granos por hilera.

El diseño experimental utilizado fue boque completo al azar con 16 híbridos y cada uno con 4 repeticiones. Los híbridos experimentales fueron: D-8008, BE-9005, E-8008, BF-9302, BF-9417, BF-9719, BG-9619, BG-9621, BG-9623, C-8008. Los híbridos comerciales fueron: INIA 611, Star, M-8480, Maximus, P30F35 y XB-8030.

Se hicieron correcciones al peso de campo llevando la humedad del grano al 14\%, luego se corrigió por fallas y por último se expresó el rendimiento de grano en $\mathrm{t} / \mathrm{ha}$.

\section{Resultados y Discusión}

Se detectaron diferencias altamente significativas entre los híbridos en estudio (Tabla 1 y Tabla 2) para todas las características evaluadas.

El coeficiente de variabilidad (CV), usado como una medida de precisión en la conducción de experimentos (Wong et al., 2007) para el rendimiento 
Tabla 1. Cuadrados medios del análisis de variancia para rendimiento de grano, altura de planta, altura de mazorca, floración masculina, floración femenina, ángulo de inserción de hoja, humedad del grano y peso de diez mazorcas.

\begin{tabular}{|c|c|c|c|c|c|c|c|c|c|}
\hline $\begin{array}{l}\text { Fuente de } \\
\text { variación }\end{array}$ & $\begin{array}{c}\text { Grados de } \\
\text { libertad }\end{array}$ & $\begin{array}{c}\text { Rendimiento } \\
\text { de grano } \\
\text { (kg/ha) }\end{array}$ & $\begin{array}{l}\text { Altura de } \\
\text { planta } \\
(\mathrm{cm})\end{array}$ & $\begin{array}{c}\text { Altura de } \\
\text { mazorca } \\
(\mathrm{cm})\end{array}$ & $\begin{array}{c}\text { Floración } \\
\text { masculina } \\
\text { (días) }\end{array}$ & $\begin{array}{l}\text { Floración } \\
\text { femenina } \\
\text { (días) }\end{array}$ & $\begin{array}{c}\text { Ángulo de } \\
\text { inserción de } \\
\text { hoja }\left(\left(^{\circ}\right)\right.\end{array}$ & $\begin{array}{l}\text { Humedad } \\
\text { del grano } \\
(\%)\end{array}$ & $\begin{array}{l}\text { Peso de diez } \\
\text { mazorcas } \\
\quad(\mathrm{kg})\end{array}$ \\
\hline Bloque & 3 & 7,29 & 948,11 & 333,65 & 23,87 & 73,50 & 18,00 & 56,68 & 0,04 \\
\hline Híbrido & 15 & $8,78^{* * *}$ & $580,19 * *$ & $375,93 * *$ & $254,50 * *$ & $80,46^{* *}$ & $111,95 * *$ & $21,92 * *$ & $0,16^{* *}$ \\
\hline Error & 45 & 1,35 & 33,44 & 28,20 & 6,55 & 6,54 & 1,80 & 6,21 & 0,01 \\
\hline Total & 63 & & & & & & & & \\
\hline Media & & 8,9 & 159,5 & 70,6 & 103,2 & 106,7 & 36,8 & 34,1 & 1,5 \\
\hline $\mathrm{CV}(\%)$ & & 12,9 & 3,6 & 7,5 & 2,4 & 2,3 & 3,6 & 7,2 & 6,5 \\
\hline
\end{tabular}

** Significancia estadística al 0,01 de probabilidad.

Tabla 2. Cuadrados medios del análisis de variancia para peso de grano de diez mazorcas, peso de cien granos, índice de mazorca, porcentaje de desgrane, longitud de mazorca, diámetro de mazorca, número de hileras y número de granos por hilera.

\begin{tabular}{|c|c|c|c|c|c|c|c|c|c|}
\hline $\begin{array}{l}\text { Fuente de } \\
\text { variación }\end{array}$ & $\begin{array}{c}\text { Grados de } \\
\text { libertad }\end{array}$ & $\begin{array}{c}\text { Peso de } \\
\text { grano de diez } \\
\text { mazorcas } \\
(\mathrm{kg})\end{array}$ & $\begin{array}{l}\text { Peso de } \\
\text { cien granos } \\
\text { (g) }\end{array}$ & $\begin{array}{l}\text { Índice de } \\
\text { mazorca }\end{array}$ & $\begin{array}{c}\text { Porcentaje } \\
\text { de desgrane } \\
(\%)\end{array}$ & $\begin{array}{c}\text { Longitud de } \\
\text { mazorca } \\
(\mathrm{cm})\end{array}$ & $\begin{array}{l}\text { Diámetro de } \\
\text { mazorca } \\
(\mathrm{cm})\end{array}$ & $\begin{array}{l}\text { Número de } \\
\text { hileras }\end{array}$ & $\begin{array}{l}\text { Número de } \\
\text { granos por } \\
\text { hilera }\end{array}$ \\
\hline Bloque & 3 & 0,03 & 0,55 & 0,06 & 1,11 & 0,36 & 0,17 & 0,05 & 4,21 \\
\hline Híbrido & 15 & $0,10 * *$ & $39,43 * *$ & $0,07 * *$ & $9,05 * *$ & $4,76^{* * *}$ & $0,23 * *$ & $7,83 * *$ & $19,08 * *$ \\
\hline Error & 45 & 0,01 & 1,73 & 0,01 & 0,36 & 0,30 & 0,01 & 0,28 & 1,35 \\
\hline Total & 63 & & & & & & & & \\
\hline Media & & 1,3 & 32,1 & 1,1 & 82,8 & 14,6 & 4,7 & 15,0 & 29,3 \\
\hline $\mathrm{CV}(\%)$ & & 6,3 & 4,1 & 8,1 & 0,7 & 3,7 & 2,5 & 3,5 & 3,9 \\
\hline
\end{tabular}

** Significancia estadística al 0,01 de probabilidad.

de grano fue $12,9 \%$, considerado como aceptable (Tabla 1). Los CV de las demás características fluctuaron entre $2,3 \%$ y $7,5 \%$, por lo tanto, los datos fueron homogéneos. Los caracteres biométricos de la mazorca como longitud de mazorca, diámetro de mazorca, número de hileras y número de granos por hilera tienen CV que varía de $2,5 \%$ a $3,9 \%$ (Tabla 2), considerados datos de poca variación.

Se observa en la Tabla 3 que el híbrido experimental D-8008 superó a los híbridos comerciales en rendimiento de grano, siendo estadísticamente similar a BF-9417, BF-9719, BG-9619, E-8008, C-8008 y BE-9005, todos experimentales también. El híbrido C-8008 tuvo menor rendimiento de grano que en otro experimento, debido a que, entre otros factores, el período de llenado de granos fue menor y, la duración de este período, está relacionada con el rendimiento de maíces híbridos (Bolaños, 1995).

El híbrido comercial M-8480 tuvo la menor altura de planta y altura de mazorca, lo que permitiría aumentar su densidad de siembra para compensar su bajo rendimiento. E-8008 es el híbrido de mayor altura de planta y alto rendimiento de grano, el que podría sembrarse a baja densidad y obtener altos rendimientos utilizando menor cantidad de semilla.

En cuanto a floración masculina y femenina, los maíces tardíos son los que tienen mayor rendimiento de grano, tal como D-8008 y BF-9417, debido al mayor tiempo que permanecen en campo (Paliwal, 2001). Además, la precocidad guarda relación con el porcentaje de humedad del grano: a mayor humedad, menor precocidad de la planta (PCIM, 1965), lo que se comprueba en los híbridos P30F35, BF-9417, BF-9719, entre otros.

Respecto del ángulo de inserción de hoja, los híbridos con alto rendimiento de grano poseen ángulos menores a $45^{\circ}$, lo que significa que las hojas superiores tienen orientación vertical, favoreciendo la distribución de la radiación solar al resto de la planta (Jugenheimer, 1981). Pendleton y Hammond, citados por Montemayor et al. (2006), observaron que maíces con hojas de tendencia vertical arriba de la mazorca, pueden ser más productivos que aquellos con hojas de tendencia horizontal. 
Tabla 3. Promedios y comparación de medias para rendimiento de grano, altura de planta, altura de mazorca, floración masculina, floración femenina, ángulo de inserción de hoja, humedad del grano y peso de diez mazorcas.

\begin{tabular}{lcccccccc}
\hline Híbrido & $\begin{array}{c}\text { Rendimiento } \\
\text { de grano } \\
\text { (t/ha) }\end{array}$ & $\begin{array}{c}\text { Altura de } \\
\text { planta } \\
(\mathrm{m})\end{array}$ & $\begin{array}{c}\text { Altura de } \\
\text { mazorca } \\
(\mathrm{m})\end{array}$ & $\begin{array}{c}\text { Floración } \\
\text { masculina } \\
(\text { días })\end{array}$ & $\begin{array}{c}\text { Floración } \\
\text { femenina } \\
(\text { días })\end{array}$ & $\begin{array}{c}\text { Ángulo de } \\
\text { inserción de } \\
\text { hoja } \\
\left({ }^{\circ}\right)\end{array}$ & $\begin{array}{c}\text { Humedad } \\
\text { de grano } \\
(\%)\end{array}$ & $\begin{array}{c}\text { Peso de diez } \\
\text { mazorcas } \\
(\mathrm{kg})\end{array}$ \\
\hline D-8008 & $10,9 \mathrm{a}$ & $1,65 \mathrm{bcd}$ & $0,85 \mathrm{a}$ & $129 \mathrm{a}$ & $106 \mathrm{defg}$ & $39,6 \mathrm{~cd}$ & $32,0 \mathrm{defg}$ & $1,53 \mathrm{ef}$ \\
BF-9417 & $10,8 \mathrm{a}$ & $1,65 \mathrm{bcd}$ & $0,71 \mathrm{~cd}$ & $104 \mathrm{bc}$ & $111 \mathrm{bc}$ & $32,9 \mathrm{hi}$ & $35,0 \mathrm{abcdef}$ & $1,70 \mathrm{bcd}$ \\
BF-9719 & $10,6 \mathrm{a}$ & $1,73 \mathrm{ab}$ & $0,81 \mathrm{ab}$ & $104 \mathrm{bc}$ & $108 \mathrm{bcde}$ & $40,6 \mathrm{c}$ & $36,5 \mathrm{abc}$ & $1,87 \mathrm{a}$ \\
BG-9619 & $10,5 \mathrm{ab}$ & $1,57 \mathrm{def}$ & $0,66 \mathrm{de}$ & $107 \mathrm{~b}$ & $109 \mathrm{bcde}$ & $36,9 \mathrm{ef}$ & $36,0 \mathrm{abcd}$ & $1,81 \mathrm{ab}$ \\
E-8008 & $10,3 \mathrm{abc}$ & $1,81 \mathrm{a}$ & $0,87 \mathrm{a}$ & $105 \mathrm{bc}$ & $107 \mathrm{cdef}$ & $45,4 \mathrm{~b}$ & $33,2 \mathrm{bcdefg}$ & $1,59 \mathrm{cdef}$ \\
C-8008 & $9,8 \mathrm{abcd}$ & $1,64 \mathrm{bcd}$ & $0,71 \mathrm{~cd}$ & $101 \mathrm{~cd}$ & $105 \mathrm{efg}$ & $28,2 \mathrm{k}$ & $35,5 \mathrm{abcde}$ & $1,37 \mathrm{gh}$ \\
BE-9005 & $9,5 \mathrm{abcde}$ & $1,62 \mathrm{cde}$ & $0,68 \mathrm{cde}$ & $104 \mathrm{bc}$ & $110 \mathrm{bcd}$ & $36,8 \mathrm{ef}$ & $36,0 \mathrm{abcd}$ & $1,70 \mathrm{bcde}$ \\
BG-9621 & $8,7 \mathrm{bcdef}$ & $1,57 \mathrm{def}$ & $0,57 \mathrm{fg}$ & $95 \mathrm{ef}$ & $99 \mathrm{~h}$ & $34,0 \mathrm{gh}$ & $33,7 \mathrm{abcdefg}$ & $1,74 \mathrm{abc}$ \\
P30F35 & $8,5 \mathrm{cdef}$ & $1,68 \mathrm{bc}$ & $0,70 \mathrm{~cd}$ & $105 \mathrm{bc}$ & $116 \mathrm{a}$ & $36,3 \mathrm{ef}$ & $37,5 \mathrm{a}$ & $1,88 \mathrm{a}$ \\
Star & $8,4 \mathrm{cdef}$ & $1,39 \mathrm{~g}$ & $0,63 \mathrm{def}$ & $101 \mathrm{~cd}$ & $105 \mathrm{efg}$ & $40,0 \mathrm{~cd}$ & $30,7 \mathrm{~g}$ & $1,51 \mathrm{fg}$ \\
M-8480 & $8,4 \mathrm{def}$ & $1,32 \mathrm{~g}$ & $0,51 \mathrm{~g}$ & $97 \mathrm{ef}$ & $101 \mathrm{~h}$ & $35,9 \mathrm{fg}$ & $31,0 \mathrm{fg}$ & $1,54 \mathrm{def}$ \\
BF-9302 & $8,2 \mathrm{def}$ & $1,58 \mathrm{def}$ & $0,69 \mathrm{cde}$ & $94 \mathrm{f}$ & $102 \mathrm{gh}$ & $30,0 \mathrm{jk}$ & $32,5 \mathrm{cdefg}$ & $1,62 \mathrm{cdef}$ \\
BG-9623 & $7,6 \mathrm{efg}$ & $1,49 \mathrm{f}$ & $0,61 \mathrm{ef}$ & $99 \mathrm{de}$ & $103 \mathrm{fgh}$ & $34,1 \mathrm{gh}$ & $36,7 \mathrm{ab}$ & $1,54 \mathrm{def}$ \\
INIA 611 & $7,5 \mathrm{fg}$ & $1,63 \mathrm{~cd}$ & $0,75 \mathrm{bc}$ & $102 \mathrm{~cd}$ & $106 \mathrm{defg}$ & $38,2 \mathrm{de}$ & $31,7 \mathrm{efg}$ & $1,18 \mathrm{i}$ \\
XB-8030 & $6,8 \mathrm{fg}$ & $1,56 \mathrm{def}$ & $0,79 \mathrm{ab}$ & $104 \mathrm{bc}$ & $112 \mathrm{~b}$ & $31,6 \mathrm{ij}$ & $36,7 \mathrm{ab}$ & $1,54 \mathrm{def}$ \\
Maximus & $6,2 \mathrm{~g}$ & $1,53 \mathrm{ef}$ & $0,69 \mathrm{cde}$ & $96 \mathrm{ef}$ & $102 \mathrm{gh}$ & $48,3 \mathrm{a}$ & $31,5 \mathrm{efg}$ & $1,25 \mathrm{hi}$ \\
\hline
\end{tabular}

Medias con la misma letra no son significativamente diferentes (prueba de Duncan al 0,05 de probabilidad).

Tabla 4. Promedios y comparación de medias para peso de grano de diez mazorcas, peso de cien granos, índice de mazorca, porcentaje de desgrane, longitud de mazorca, diámetro de mazorca, número de hileras y número de granos por hilera.

\begin{tabular}{|c|c|c|c|c|c|c|c|c|}
\hline Híbrido & $\begin{array}{c}\text { Peso de } \\
\text { grano de diez } \\
\text { mazorcas } \\
(\mathrm{kg})\end{array}$ & $\begin{array}{l}\text { Peso de cien } \\
\text { granos } \\
(\mathrm{g})\end{array}$ & $\begin{array}{l}\text { Índice de } \\
\text { mazorca }\end{array}$ & $\begin{array}{c}\text { Porcentaje de } \\
\text { desgrane } \\
(\%)\end{array}$ & $\begin{array}{l}\text { Longitud de } \\
\text { mazorca } \\
(\mathrm{cm})\end{array}$ & $\begin{array}{l}\text { Diámetro de } \\
\text { mazorca } \\
(\mathrm{cm})\end{array}$ & $\begin{array}{l}\text { Número de } \\
\text { hileras }\end{array}$ & $\begin{array}{l}\text { Número de } \\
\text { granos por } \\
\text { hilera }\end{array}$ \\
\hline D-8008 & 1,30 cde & $30,3 \mathrm{bcd}$ & $1,37 \mathrm{a}$ & $84,8 \mathrm{~b}$ & $13,9 \mathrm{de}$ & $4,6 \mathrm{de}$ & $16,3 \mathrm{~b}$ & $28,7 \mathrm{def}$ \\
\hline BF-9417 & $1,39 \mathrm{~cd}$ & 29,8 cde & $1,35 \mathrm{ab}$ & $81,7 \mathrm{fg}$ & $14,1 \mathrm{de}$ & $4,9 \mathrm{abc}$ & $16,7 \mathrm{ab}$ & 29,5 bcde \\
\hline BF-9719 & $1,57 \mathrm{a}$ & $32,0 \mathrm{~b}$ & $1,05 \mathrm{def}$ & $83,9 \mathrm{bc}$ & $16,4 \mathrm{a}$ & $4,9 \mathrm{abc}$ & $17,3 \mathrm{a}$ & $31,4 a b$ \\
\hline BG-9619 & $1,52 \mathrm{ab}$ & $29,1 \mathrm{de}$ & 1,12 cde & $84,3 \mathrm{bc}$ & $15,1 \mathrm{bc}$ & $4,8 \mathrm{bcd}$ & $17,2 \mathrm{a}$ & $31,8 \mathrm{a}$ \\
\hline E-8008 & 1,33 cde & $32,1 \mathrm{~b}$ & $1,15 \mathrm{~cd}$ & $83,9 \mathrm{bc}$ & $14,4 \mathrm{~cd}$ & $4,7 \mathrm{~cd}$ & $16,1 \mathrm{~b}$ & 28,1 def \\
\hline C-8008 & $1,13 \mathrm{fg}$ & $26,7 \mathrm{f}$ & $1,22 \mathrm{bc}$ & $82,9 \mathrm{de}$ & $13,8 \mathrm{de}$ & 4,5 ef & $15,1 \mathrm{c}$ & $31,0 \mathrm{ab}$ \\
\hline BE-9005 & $1,40 \mathrm{bc}$ & $35,2 \mathrm{a}$ & $1,15 \mathrm{~cd}$ & 82,4 ef & $16,7 \mathrm{a}$ & $4,4 \mathrm{f}$ & $13,7 \mathrm{e}$ & $30,8 \mathrm{ab}$ \\
\hline BG-9621 & $1,41 \mathrm{bc}$ & $36,5 \mathrm{a}$ & $0,95 \mathrm{f}$ & $81,0 \mathrm{~g}$ & $15,0 \mathrm{bc}$ & $4,8 \mathrm{~cd}$ & 14,4 cde & 27,8 ef \\
\hline P30F35 & $1,54 \mathrm{a}$ & $35,3 \mathrm{a}$ & 0,97 ef & $81,7 \mathrm{fg}$ & $15,4 \mathrm{~b}$ & $5,1 \mathrm{a}$ & $14,7 \mathrm{~cd}$ & $31,7 \mathrm{a}$ \\
\hline Star & 1,30 cde & $31,4 b c$ & $1,07 \mathrm{cdef}$ & $85,9 \mathrm{a}$ & $15,1 \mathrm{bc}$ & 4,5 ef & $13,9 \mathrm{de}$ & $30,7 \mathrm{abc}$ \\
\hline M-8480 & 1,25 ef & $30,3 \mathrm{bcd}$ & 1,12 cde & $81,2 \mathrm{~g}$ & $15,1 b c$ & 4,5 ef & $13,8 \mathrm{e}$ & $31,7 \mathrm{a}$ \\
\hline BF-9302 & 1,33 cde & $35,9 \mathrm{a}$ & 0,97 ef & $81,8 \mathrm{fg}$ & 13,3 ef & $4,9 \mathrm{abc}$ & 14,2 cde & $27,1 \mathrm{fg}$ \\
\hline BG-9623 & $1,26 \mathrm{def}$ & $35,6 \mathrm{a}$ & $1,05 \mathrm{def}$ & $81,6 \mathrm{fg}$ & $13,6 \mathrm{def}$ & $5,0 a b$ & $14,7 \mathrm{~cd}$ & $26,1 \mathrm{gh}$ \\
\hline INIA 611 & $0,99 \mathrm{~h}$ & $28,0 \mathrm{ef}$ & $1,35 \mathrm{ab}$ & $83,7 \mathrm{~cd}$ & $13,8 \mathrm{de}$ & $4,1 \mathrm{~g}$ & $13,8 \mathrm{e}$ & 28,9 cde \\
\hline XB-8030 & $1,25 \mathrm{ef}$ & $35,3 \mathrm{a}$ & $1,07 \mathrm{cdef}$ & $80,8 \mathrm{~g}$ & $15,6 \mathrm{~b}$ & $4,7 \mathrm{~cd}$ & $12,7 \mathrm{f}$ & $29,8 \mathrm{bcd}$ \\
\hline Maximus & $1,04 \mathrm{gh}$ & 30,1 bcde & $1,02 \mathrm{def}$ & $83,5 \mathrm{~cd}$ & $12,8 \mathrm{f}$ & 4,5 ef & $16,1 \mathrm{~b}$ & $24,6 \mathrm{~h}$ \\
\hline
\end{tabular}

Medias con la misma letra no son significativamente diferentes (prueba de Duncan al 0,05 de probabilidad).

La Tabla 4 muestra las características que más contribuyen al rendimiento de grano. El híbrido D-8008 presentó el mayor índice de mazorca con 1.3 mazorcas, siendo similar a los híbridos INIA 611 y BF-9417. Esta es una de las características que más aporta a su rendimiento, junto con el porcentaje de desgrane y el número de hileras de la mazorca. Los híbridos BF-9417, BF-9719 y BG-9619 deben su alto rendimiento, principalmente, al alto peso de mazorcas y peso de granos, tal como se aprecia en la Tabla 3 y Tabla 4, respectivamente.

En cuanto al peso de cien granos, los híbridos más rendidores tienen bajos valores, es decir, tienen semillas pequeñas. Solo un híbrido que obtuvo alto valor, BE-9005, rindió 9,52 t/ha, resultado similar al que tuvo el híbrido D-8008 (Tabla 3). Al parecer 
la característica que mejor se relaciona con el rendimiento es el peso de mil granos (Wong et al., 2007; Ferraris y Couretot, 2004 y Rivetti, 2006).

Una de las características que más aportaron al rendimiento de los híbridos D-8008 y BF-9417 fue el índice de mazorca. Vásquez et al. (2003) encontraron una correlación positiva entre el rendimiento de grano y la prolificidad, expresada en el índice de mazorca. Torres y Rodríguez (1993) indican que el componente del rendimiento que más influencia tiene en la mayor producción es la prolificidad.

Respecto del porcentaje de desgrane, el híbrido comercial Star presentó un valor de $85,9 \%$, siendo superior a los híbridos experimentales, sin embargo, ocupó el décimo lugar en rendimiento (Tabla 3), lo que supone que un alto desgrane no implica un alto rendimento, ya que sería más importante la cantidad de mazorcas por planta y características biométricas de la mazorca. No obstante, se observa que todos los híbridos superan el $80 \%$ de desgrane, valor deseable en este tipo de maíces (Bocanegra, 1953).

Los híbridos BF-9719 y BE-9005 superaron a los híbridos comerciales en longitud de mazorca (Tabla 4). Además tuvieron altos rendimientos, similares al híbrido D-8008. Una de las pocas características en las que destacó un híbrido comercial fue en el diámetro de la mazorca, donde P30F35 fue superior a los híbridos experimentales. Se observa también que el híbrido BF-9719 debe parte de su alto rendimiento a valores superiores de longitud y diámetro de mazorca. Wong et al. (2007) observaron que el diámetro de mazorca y la longitud de mazorca contribuyen a aumentar el número de granos por mazorca por unidad de superficie y, por tanto, el rendimiento.

Los datos biométricos de la mazorca son los que más contribuyen al rendimiento de maíces híbridos (Jugenheimer, 1981), lo que se comprueba en el híbrido BF-9719, que presenta los más altos valores de longitud, diámetro, número de hileras y número de granos por hilera de la mazorca (Tabla 4). Estas características influyeron en su alto rendimiento de la siguiente manera: un alto valor de diámetro permitió un mayor número de hileras, la mayor longitud de mazorca influyó en el alto número de granos por hilera, todo ello implica un mayor número de granos por planta y por unidad de superficie.

El híbrido BF-9417 debió su alto rendimiento a las siguientes características: índice, diámetro y número de hileras de la mazorca, mientras que en el híbrido BG-9619 destacaron el peso de grano de diez mazorcas, el número de hileras y el número de granos por hilera como contribuyentes a su rendimiento de grano.

\section{Conclusiones}

El híbrido que tuvo mayor rendimiento de grano fue $\mathrm{D}-8008$ con 10,9 t/ha y presentó el mayor índice de mazorca con 1,3 mazorcas.

Los híbridos comerciales M-8480 y Star tuvieron la menor altura de planta con $1,32 \mathrm{~m}$ y $1,39 \mathrm{~m}$. La menor altura de mazorca fue para los híbridos M-8480 y BG-9621 con 0,51 m y 0,57 m.

Los híbridos más precoces fueron BF-9302, BG-9621, Maximus y M-8480 con 94, 95, 96 y 97 días a la floración masculina. Los mismos híbridos tuvieron los menores valores de humedad del grano: $32,5 \%, 33,7 \%, 31,5 \%$ у $31 \%$.

El menor ángulo de inserción de hoja fue para el híbrido C- 8008 con $28,2^{\circ}$, el cual fue similar al híbrido BF-9302, con $30^{\circ}$.

El híbrido con mayor peso de diez mazorcas fue P30F35 con $1,88 \mathrm{~kg}$. Asimismo el mayor peso de grano de diez mazorcas fue para el híbrido BF9719 con 1,57 kg. El híbrido BG-9621 con 36,5 g tuvo el mayor peso de cien granos.

El mayor porcentaje de desgrane le corresponde al híbrido Star con 85,9\%, seguido de los híbridos: D-8008, BG-9619, E-8008 y BF-9719 con $84,8 \%$, $84,3 \%, 83,9 \%$ y $83,9 \%$.

El híbrido que tuvo la mayor longitud de mazorca fue BE-9005 con $16,7 \mathrm{~cm}$. El mayor diámetro de mazorca fue para el híbrido P30F35 con 5,09 cm. El mayor número de hileras fue para el híbrido BF-9719 con 17,3 hileras y el mayor número de granos por hilera fue para el híbrido BG-9619 con 31,8 granos.

Uno de los híbridos más prometedores respecto de rendimiento de grano, sus componentes y características favorables fue BF-9719, ya que sobresalió en rendimiento de grano, ángulo de inserción de hoja, peso de grano de diez mazorcas, longitud y diámetro de mazorcas, número de hileras y número de granos por hilera. BF-9417 fue otro híbrido experimental que destacó en rendimiento, ángulo de inserción de hoja, índice de mazorca, diámetro de mazorca y número de hileras. 


\section{Literatura Citada}

Beingolea, L.; Manrique, A.; Fegan, W.; Sánchez, H.; Noriega, V.; Borbor, M.; Chura, J.; Castillo, J. y Sarmiento, J.

1993. Manual del maíz para la Costa. Primera Edición. Publicación de la Coordinación General de la Actividad Difusión de Tecnología del Proyecto TTA. Lima, Perú. 93 pp.

Bocanegra, S.

1953. El maíz híbrido en la costa peruana. Programa Cooperativo Experimental Agropecuario. Ministerio de Agricultura. Lima, Perú. 45 pp.

Bolaños, J.

1995. Physiological bases for yield differences in selected maize cultivars from Central America. Field Crops, 42: 69-80.

Chura, J. y Sevilla, R.

2002. Mejoramiento genético del maíz en el Perú. Simposium: El mejoramiento genético de las plantas en el Perú. Primera Edición. Sociedad Peruana de Genética. Lima, Perú. 189 pp.

Ferraris, G. y Couretot, L.

2004. Ensayo comparativo de híbridos comerciales de maíz en el área de Colón-Wheelwright, Argentina. Maíz. Resultados de las Unidades Demostrativas. Desarrollo Rural INTA Pergamino. Proyecto Regional Agrícola, CERBAN, pp. 37-43.

Jugenheimer, R.W.

1981. Maíz: variedades mejoradas, métodos de cultivo y producción de semillas. Primera Edición. Editorial Limusa. México. 840 pp.

Ministerio de Agricultura.

Sector Agrario. Cultivos de Importancia Nacional (http:// www.portalagrario.gob.pe/portal/sector-agrario/agricola/ cultivos-de importancia-nacional; 20 de diciembre de 2012).

Ministerio de Agricultura-Oficina General de Planificación Agraria y Dirección General de Promoción Agraria.

Plan Estratégico de la Cadena Productiva de Maíz Amarillo Duro Avícola, Porcícola. (http://www.planeamientoygestion. com.pe/consultoria/images/stories/herramientas/documentos/ maz_amarillo_duro.pdf; 15 de noviembre de 2011).

Montemayor, J.; Zermeño, A.; Olague, J.; Aldaco, R.; Fortis,

M.; Salazar, E.; Cruz, J. y Vázquez-Vázquez, C.

2006. Efecto de la densidad y estructura del dosel de maíz en la penetración de la radiación solar. Revista Internacional de Botánica Experimental, 55 (75): 47-53.
Organización de las Naciones Unidas para la Alimentación y la Agricultura.

Estadísticas. FAOSTAT - Producción Agrícola. (http:/www. fao.org/corp/statistics/es/; 2 de febrero de 2013).

Paliwal, R.L.

2001. El maíz en los trópicos. Mejoramiento y producción. Colección FAO: Producción y protección vegetal № 28 . Roma. 350 pp.

Programa Cooperativo de Investigaciones en Maíz.

1965. Prueba de híbridos comerciales de maíz en Costa Norte. Universidad Nacional Agraria La Molina. Lima, Perú. $43 \mathrm{pp}$.

Rivetti, A. R.

2006. Producción de maíz bajo diferentes regímenes de riego complementario en Río Cuarto, Córdoba, Argentina. I. Rendimiento en grano de maíz y sus componentes. Revista de la Facultad de Ciencias Agrarias UNCUYO 38 (2): 25-36.

Salhuana, W. y Scheuch, F.

2004. Programa Cooperativo de Investigaciones en Maíz (PCIM): Logros y perspectivas. $50^{\circ}$ Aniversario. Editorial Universidad Nacional Agraria La Molina. Lima, Perú. $478 \mathrm{pp}$.

Sevilla, R.

2000. Perspectivas del cultivo de maíz en el Perú. El autoabastecimiento del maíz amarillo duro. Revista Agroenfoque, 15 (111): 10-12.

Torres, C. y Rodríguez, A.

1993. Evaluación de las diferencias de rendimiento entre híbridos y variedades de maíz (Zea mays L.). XXXIX Reunión Anual de la Sociedad del Programa Cooperativo Centroamericano para el Mejoramiento de Cultivos y Animales. Guatemala. 365 pp.

Vásquez, V.; Medina, A. y Paredes, J.

2003. Ensayos de híbridos de maíz (Zea mays L.) tropicales de grano amarillo de madurez precoz en Paiján, La Libertad. Revista Caxamarca, 11 (2): 45-47.

Wong, R.; Gutiérrez del Río, E.; Palomo, A.; Rodríguez, S.; Córdova, H.; Espinoza, A. y Lozano, J.

2007. Aptitud combinatoria de componentes del rendimiento en líneas de maíz para grano en la Comarca Lagunera, México. Revista Fitotecnia Mexicana, 30 (002): 181-189. 